\title{
Optimization of K-Edge Subtraction Imaging Using a Pixellated Spectroscopic Detector
}

\author{
Silvia Pani, Sarene C. Saifuddin, Filipa I.M. Ferreira, James W. Scuffham, Philipp Stratmann, Matthew D. Wilson, \\ Matthew C Veale, Steven Bell, Paul Seller, Paul J Sellin, Robert J Cernik
}

\begin{abstract}
Conventional K-edge subtraction imaging is based around the acquisition of two separate images at energies respectively below and above the K-edge of a contrast agent. This implies increased patient dose with respect to a conventional procedure and potentially incorrect image registration due to patient motion.

We present results obtained with a pixellated spectroscopic CdTe detector. A spectroscopic detector allows simultaneous acquisition of the two images by integrating appropriate bands from the transmitted $X$-ray spectrum, thus removing the above limitations; the photon counting capability of the detector limits the noise to statistical noise, thus minimizing the dose for a given signal-to-noise ratio.

Furthermore, an appropriate choice of the integration bands allows optimization of image quality, resulting from a trade-off between background removal (maximum with a narrow band) and low statistical noise (achieved with a broad band).

Results obtained with a custom test-object, simulating breast structures, and with an iodine-based contrast agent, are presented for two different image subtraction algorithms: logarithmic subtraction and dual-energy linear combination. Whilst being conceptually simpler, logarithmic subtraction is strongly dependent upon the position and width of the band selected, while linear combination allows better background removal even with a broad energy band, and therefore better image quality.
\end{abstract}

\section{INTRODUCTION}

$\mathrm{C}$ ONTRAST agent X-ray imaging increases the visibility of an anatomical region by artificially increasing its contrast with the injection of a contrast agent with higher attenuation coefficient than biological tissue. This is particularly useful in mammography, where the intrinsically low contrast of tumour against healthy tissue often prevents detection. However, the detection of small structures is often prevented by the presence

Manuscript received November 16, 2012. (Write the date on which you submitted your paper.) This work was supported by EPSRC (grant EP/H046577/1). S Saifuddin is supported by the Ministry of Health Malaysia.

S Pani is with the Department of Physics, University of Surrey, Guildford, GU2 7KH, UK (e-mail s.pani@surrey.ac.uk)

S. Saifuddin, F Ferreira, P Sellin are with the Department of Physics, University of Surrey, Guildford, GU2 7KH, UK.

J Scuffham is with the Nuclear Medicine Department, Royal Surrey County Hospital, Guildford, UK.

M. Wilson, M. Veale, S. Bell and P. Seller are with STFC Rutherford Appleton Laboratories, Didcot, UK.

P. Stratmann was with the Department of Physics, University of Surrey, Guildford, GU2 7XH, UK and is with RWTH Aachen, Germany. of a cluttered background. The background can be removed by means of K-edge subtraction, relying on the use of a contrast agent with a K-edge in an appropriate energy range. The technique, although initially proposed in the 1950s [1], has been more intensively used in recent years when digital detectors became more easily available [2], [3].

Two images are acquired, with X-ray beams of energy below and above the K-edge, respectively, and combined, typically by logarithmic subtraction, leaving an image where the background is removed, highlighting the distribution of the contrast agent.

The limitation of this approach lies in the need for a double exposure, implying increased dose to the patient and artifacts from patient motion. The present work has been carried out with a pixellated spectroscopic CdTe detector, allowing images at different energies to be acquired simultaneously and obtained by simply integrating different bands of the spectrum pixel by pixel.

We compare here the results obtained with simple logarithmic subtraction and with a more generic dual energy algorithm.

\section{MATERIALS AND METHODS}

\section{A. The detector}

The HEXITEC collaboration has developed a pixellated CdTe detector with spectroscopic capability [4]. The detector consists of an 80x80 array of pixels with $250 \mu \mathrm{m}$ pitch and 1 $\mathrm{mm}$ thickness, which grants $100 \%$ absorption efficiency in the energy range used for the present application. The device has been used for a number of applications and techniques, including nuclear medicine, X-ray diffraction, X-ray fluorescence for both medical applications and material studies.

The energy resolution of the detector is $800 \mathrm{eV}$ (FWHM) at $59.5 \mathrm{keV}$.

The detector is read out with a rate of $\sim 10^{4}$ frames/second using a framegrabber card and customized software. It was biased at $-500 \mathrm{~V}$ and, to avoid polarization, the bias was refreshed to $0 \mathrm{~V}$ for $2 \mathrm{~s}$ every $60 \mathrm{~s}$.

\section{B. Experimental set up}

A custom test object, shown in Fig. 1, was developed, consisting of a $4 \mathrm{~cm}$-thick Perspex box with $5 \mathrm{~mm}$-thick walls, 
filled with plastic spheres of different diameters (simulating the glandular component of the breast) and with olive oil (simulating the adipose component of the breast).

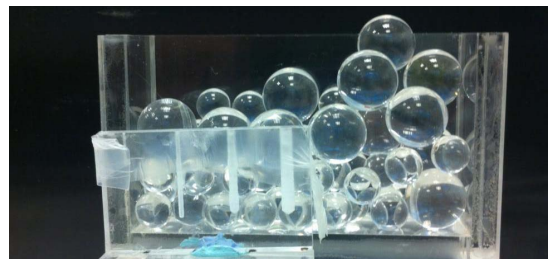

Fig 1. test object used for K-edge subtraction imaging.

A 6-mm thick Perspex slab, with cylindrical holes $3 \mathrm{~mm}, 2$ $\mathrm{mm}$ and $1 \mathrm{~mm}$ in diameter, respectively, was superimposed to the box. The holes were filled with undiluted Niopam 150 (Bracco, UK), an iodine-based contrast agent with an iodine concentration of $150 \mathrm{mg} / \mathrm{ml}$. The detector was placed at $75 \mathrm{~cm}$ from a $\mathrm{W}$-anode $\mathrm{X}$-ray source operated at $50 \mathrm{kVp}$. The spectrum of the source was modulated using $3 \mathrm{~mm} \mathrm{Al}$ in order to have the maximum intensity of $\mathrm{X}$-rays around the $\mathrm{K}$-edge of iodine $(33 \mathrm{keV})$ and hence reduce the dose from spectral components not contributing to image formation.

The tube was operated at $5 \mu \mathrm{A}$, and the mean glandular dose to the phantom was calculated as $32 \mu \mathrm{Gy}$ for the images displayed here, which is approximately 50 times lower than typical entrance doses in single-image mammography [5].

Images of extended objects were obtained by scanning the object using a remote-controlled linear stage (Micos VT-80 with a Corvus-eco controller; Micos Gmbh, Germany).

\section{Data acquisition and processing}

After calibration using characteristic X-rays from a variable energy X-ray source in the range $17.5 \mathrm{keV}$ (Mo K $\alpha$ line) to $59.5 \mathrm{keV}(\mathrm{Am}-241 \gamma)$, the spectra from all pixels were linearly interpolated in order to use the same calibration coefficients for all pixels. This allowed the full exploitation of the intrinsic energy resolution of the detector, allowing, in particular, the selection of integration bands closer to the physical K-edge of iodine.

Integration bands of varying width were chosen above and below the K-edge of iodine, respectively, as shown in Fig. 2.

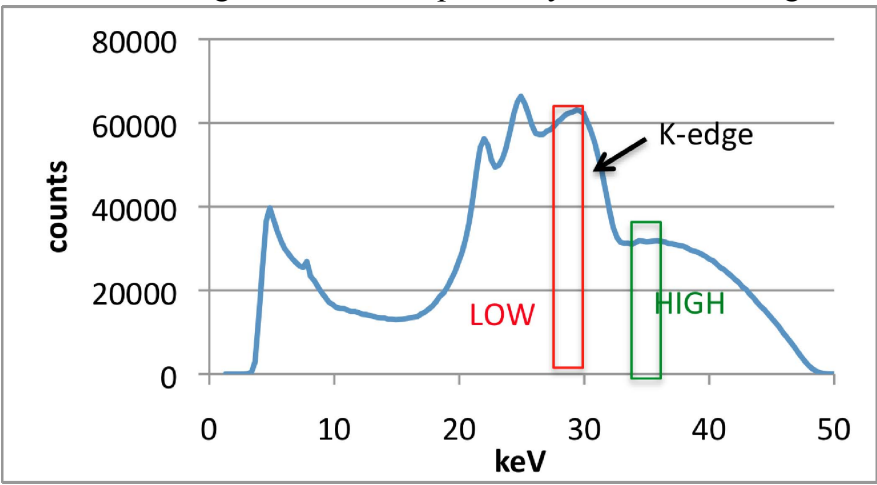

Fig. 2. Spectrum obtained by summing the spectra from pixels behind $3 \mathrm{~mm}$ of iodine, showing the selection of integration bands for K-edge subtraction imaging.

\section{Subtraction algorithms}

The first subtraction algorithm used was logarithmic subtraction. The subtracted image is calculated as

$$
\begin{aligned}
& S(i, j)=\ln \left(I_{\text {high }}^{0} / I_{\text {high }}(i, j)\right)-\ln \left(I_{\text {low }}^{0} / I_{\text {low }}(i, j)\right) \\
& =\mu_{\text {high }}^{I} x_{I}+\mu_{\text {high }}^{b g} x_{b g}-\left(\mu_{\text {low }}^{I} x_{I}+\mu_{\text {low }}^{b g} x_{b g}\right)
\end{aligned}
$$

where $I^{0}$ is the incident intensity, $\mu^{I}$ is the attenuation coefficient of iodine, $\mu^{b g}$ is the attenuation coefficient of the background, $x_{I}$ is the iodine thickness, $x_{b g}$ is the thickness of the background region, and the subscripts high, low refer to the high energy image and the low energy image, respectively.

If the two integration bands are sufficiently close to each other, the difference between $\mu^{b g}$ high and $\mu_{\text {low }}^{\text {bg }}$ becomes negligible, and equation (1) becomes

$S(i, j) \approx\left(\mu_{\text {high }}^{I}-\mu_{\text {high }}^{I}\right) x_{I}$

This algorithm clearly relies strongly on the choice of narrow and close to each other integration bands, and the contrast is the higher the closer they are, so that the maximum difference in the attenuation coefficients of iodine on the two sides of the K-edge is exploited.

A more generic algorithm is the dual energy algorithm initially proposed by Lehmann et al [6] and subsequently used in several K-edge imaging applications [7], [8]. It consists of decomposing an image into two components; for the present case, the components chosen were iodine, representing the contrast agent, and water, representing the background materials. This algorithm doesn't rely specifically on the presence of a K-edge, but on the presence of sufficient variations in the attenuation coefficients of the two materials at the two energies chosen. The two equations describing the attenuation at the two energies can be written as

$$
\begin{aligned}
& \ln \left(I_{0} / I\right)_{\text {low }}=\frac{\mu}{\rho}_{\text {low }}^{I}(\rho x)_{I}+\frac{\mu}{\rho}{ }_{\text {low }}^{\text {water }}(\rho x)_{\text {water }} \\
& \ln \left(I_{0} / I\right)_{\text {high }}=\frac{\mu}{\rho}_{\text {high }}^{I}(\rho x)_{I}+\frac{\mu}{\rho}_{\text {high }}^{\text {water }}(\rho x)_{\text {water }}
\end{aligned}
$$

where $\rho$ is the density of each material and $\mu / \rho$ is the mass attenuation coefficients for the two materials at the two energies used.

This system of linear equations can be solved for $(\rho x)_{I}$ and $(\rho x)_{\text {water }}$ giving an "iodine equivalent" image and a "water equivalent" image.

In the present work, the mass attenuation coefficients were chosen at the centre of each integration band. The data were sourced from XMudat [9].

The effect of the two algorithms on the visibility of details was measured using the contrast-to-noise ratio $(C N R)$ :

$$
C N R=\left(S_{\text {in }}-S_{\text {out }}\right) / \sigma_{\text {out }}
$$

where $S_{i n}$, $S_{\text {out }}$ are the average signal intensities inside and outside a detail region, respectively, and $\sigma_{\text {out }}$ is the standard deviation of the region outside the detail. 


\section{RESULTS AND DISCUSSION}

Fig. 3 and Fig 4 show comparison of the log-subtracted image and of the iodine component obtained with the dual energy algorithm for two different integration bands.

It is apparent from the figures that, while both algorithms allow good detail visibility with an integration band of $2 \mathrm{keV}$, the logarithmic subtraction performs poorly for a wide integration band.
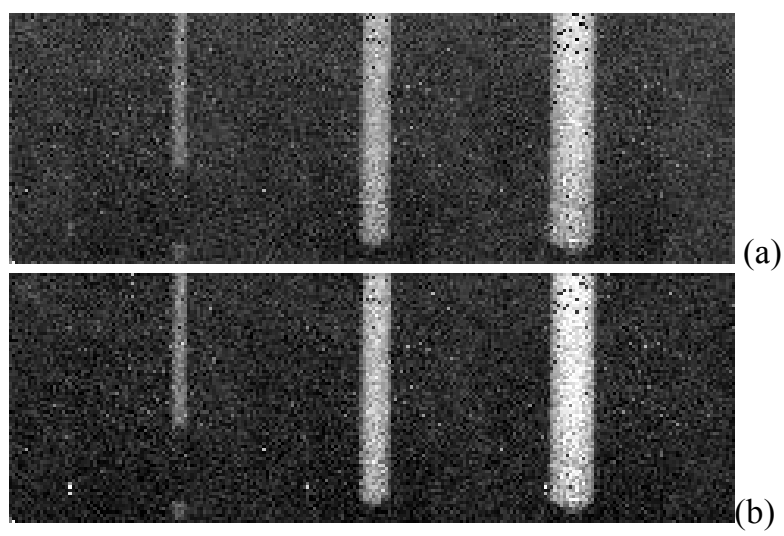

b)

Fig. 3. Comparison of the log-subtracted image (a) and of the iodine
mponent obtained with the dual-energy algorithm (b) with an integration band width of $2 \mathrm{keV}$.

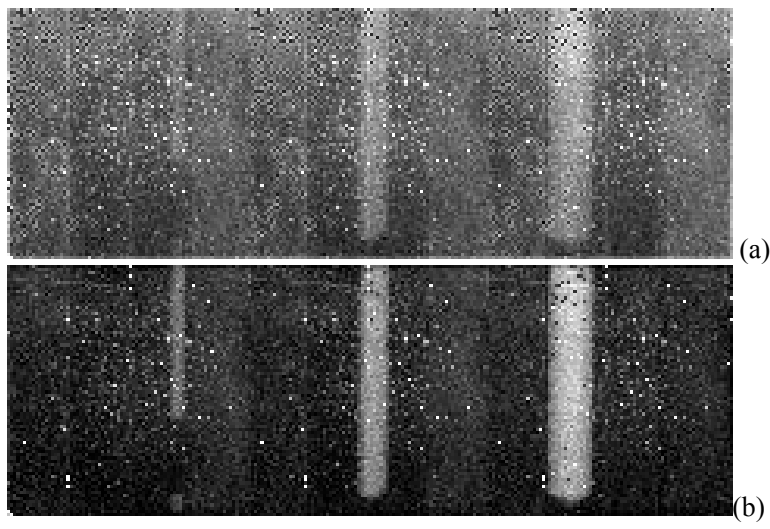

Fig. 4. Comparison of the log-subtracted image (a) and of the iodine component obtained with the dual-energy algorithm (b) with an integration band width of $17 \mathrm{keV}$.

This is confirmed by the plots shown in Fig. 5, displaying the contrast-to-noise ratio measured for the three details as a function of the integration bandwidth for the two algorithms.

All plots have maxima between 2 and $5 \mathrm{keV}$. The optimal integration band results from a compromise between low statistical noise, achieved with wide integration bands, and high contrast, achieved with narrow bands, which exploit the maximum difference in the attenuation coefficients of iodine on the two sides of the K-edge.

It is apparent from the figures that the dual energy algorithm performs consistently better than logarithmic subtraction. This is due to a number of reasons.

Firstly, as mentioned, this algorithm is less reliant on the selection of two integration bands that are close to each other; therefore, the drop in contrast resulting from the use of broader bands, for which the difference in the attenuation coefficient of iodine decreases, is less strong for the dual energy algorithm.

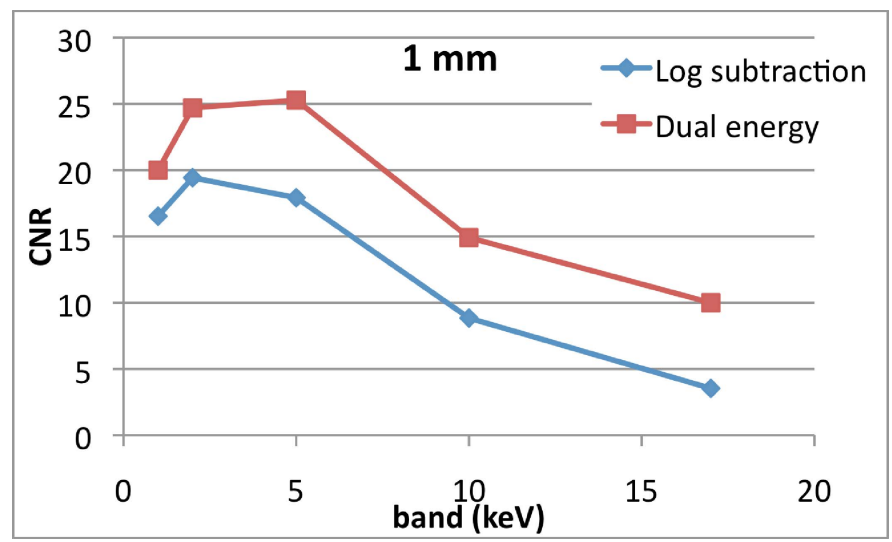

(a)

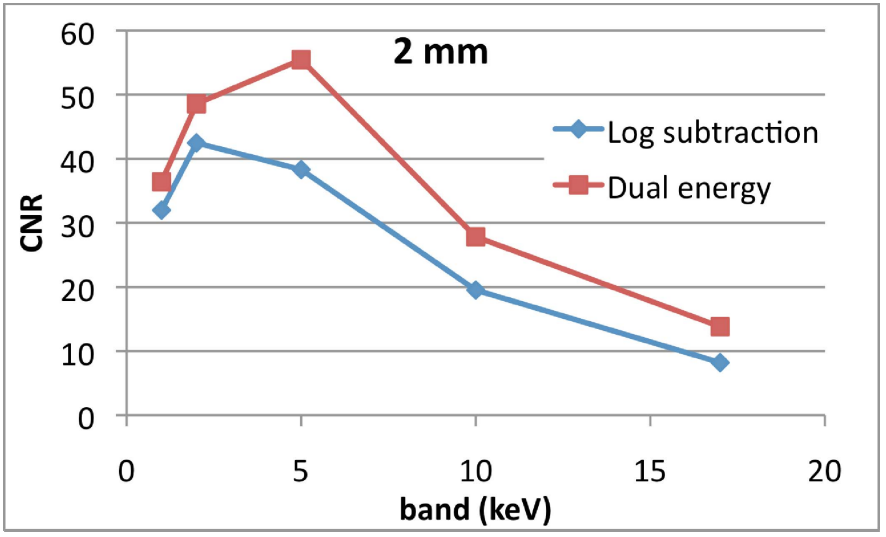

(b)

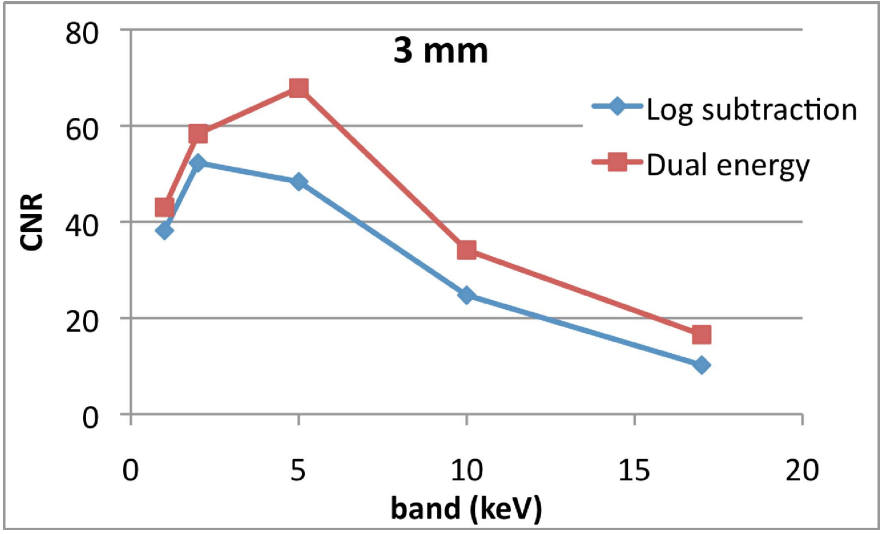

(c)

Fig. 5. Comparison of the contrast-to-noise ratio measured on the 1-mm detail (a), the 2-mm detail (b) and the 3-mm detail (c) on the images obtained with the two algorithms.

Secondly, the logarithmic subtraction algorithm relies on the strong assumption that the variation between the attenuation coefficients of iodine at the two energies is negligible. When the average energies of the two bands are apart, this assumption no longer holds, and the background structures are no longer completely removed from the subtracted image, adding to the structural noise in the image.

The better capability of the dual energy algorithm for background removal also accounts for the fact that the 
maximum of the CNR plot lies at a higher energy for this algorithm than for the logarithmic subtraction: with less dependency upon structural noise, the benefits resulting from increased statistics are predominant.

Fig. 6 shows the water component of the object as obtained with the dual energy algorithm with integration bands of 2 $\mathrm{keV}$ and $17 \mathrm{keV}$, respectively.

In this case the superior performance of choosing narrow integration bands is apparent. In particular, the water component of the solution inside the tubes is incorrectly represented at $17 \mathrm{keV}$.

However, this shows the potential of the algorithm, at least at narrow integration bands, to obtain an image similar to conventional mammographic images. This is important for benchmarking of the technique against established ones.



(a)

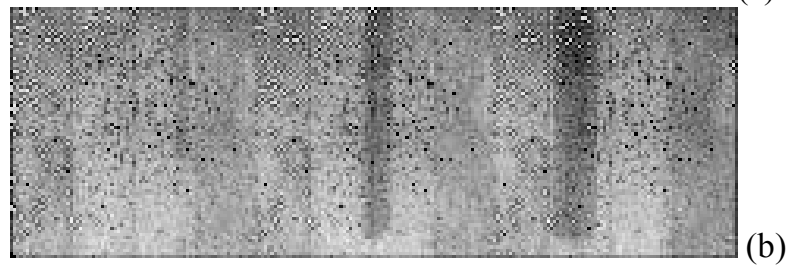

Fig. 6. Comparison of the water component of the object as obtained from the dual energy algorithm with $2 \mathrm{keV}$ integration bandwidth (a) and $17 \mathrm{keV}$ integration bandwidth (b). The dark band to the right of the images is a region outside the 6-mm Perspex slabs where the details are inserted. The bright dots correspond to noisy pixels in the device used.

\section{CONCLUSIONS AND FUTURE WORK}

Results so far proved the effectiveness of K-edge subtraction imaging in background removal to highlight the distribution of a contrast agent with doses significantly lower than standard mammographic dose. A dual-energy algorithm, relying on less stringent assumptions, performs consistently better than simple logarithmic subtraction in terms of both contrast improvement and structural noise reduction.

The technique could be used in the future to assess vascularisation around tumours, or uptake/washout curves of a contrast agent (the latter to be measured on a dynamic test object) as an indicator of malignancy. The reduction in dose suggests the feasibility of the technique as a screening method for at least part of the population: for instance, young women with a family history of breast cancer, for which conventional mammography may be inconclusive due to the high fraction of glandular tissue, limiting the visibility of tumours, at a young age.

In the near future, more complex subtraction algorithms will be implemented, taking into account the variation of the attenuation coefficients of the materials across the energy bands selected and corrections for loss of linearity in the detector response when higher X-ray tube currents are applied, thus allowing faster image acquisition.

\section{REFERENCES}

[1] B. Jacobson 1953 "Dichromatic absorption radiographydichromography" Acta Radiol. 39: 437-52.

[2] K.L. Houk et al. 1979. "Real time digital K-edge subtraction fluoroscopy". Investigative Radiology 14(4):270-278.

[3] J.M. Lewin et al 2003. "Dual-energy contrast-enhanced digital subtraction mammography: feasibility". Radiology 229: 261-268.

[4] P. Seller et al 2011 "Pixellated $\mathrm{Cd}(\mathrm{Zn}) \mathrm{Te}$ high-energy X-ray instrument". J Inst 6: C12009.

[5] R. L. Krueger and B. A. Schueler 2001. "A survey of clinical factors and patient dose in mammography". Med Phys 28: 1449-1454.

[6] L. A. Lehmann et al 1981. "Generalized image combination in dual $\mathrm{kVp}$ digital radiography". Med Phys 8: 659-667.

[7] A. Sarnelli et al. 2007. "Quantitative analysis of the effect of energy separation in k-edge digital subtraction imaging". Phys Med Biol 52: 3015-3026.

[8] P. V. Granton et al. 2008. "Implementation of dual- and triple-energy cone-beam micro-CT for postreconstruction material decomposition". Med Phys 35: 5030-5042.

[9] R. Nowotny 1998. "XMuDat: Photon attenuatuon data on PC". IAEA Report IAEA-NDS-195. 